Europhysics Letters

PREPRINT

\title{
Nonlinear viscoelasticity of metastable complex fluids
}

\author{
Kunimasa Miyazaki $^{1}$, Hans M. Wyss ${ }^{2}$, David A. Weitz ${ }^{2}$ and David R. Reich- \\ MAN $^{1}$ \\ 1 Department of Chemistry, Columbia University, 3000 Broadway, New York, NY 10027 \\ 2 Department of Physics \& DEAS, Harvard University, Cambridge, MA 02138
}

PACS. 64.70.Pf - Glass transitions.

PACS. 83.60.Rs - Shear thinning and shear thickening.

PACS. 83.80.Hj - Suspensions, dispersions, pastes, slurries, colloids.

\begin{abstract}
Many metastable complex fluids such as colloidal glasses and gels show distinct nonlinear viscoelasticity with increasing oscillatory-strain amplitude; the storage modulus decreases monotonically as the strain amplitude increases whereas the loss modulus has a distinct peak before it decreases at larger strains. We present a qualitative argument to explain this ubiquitous behavior and use mode coupling theory (MCT) to confirm it. We compare theoretical predictions to the measured nonlinear viscoelasticity in a dense hard sphere colloidal suspensions; reasonable agreement is obtained. The argument given here can be used to obtain new information about linear viscoelasticity of metastable complex fluids from nonlinear strain measurements.
\end{abstract}

Rheological measurements are commonly used to investigate dynamical and structural properties of complex fluids. Oscillatory linear viscoelastic experiments measure the response function, or the complex modulus, $G^{*}(\omega)=G^{\prime}(\omega)+i G^{\prime \prime}(\omega)$ [1]. This provides information about the relaxation of the microstructure of the system as a function of oscillation frequency, $\omega$. This function is strictly defined only within the linear response regime, and thus an important practical experimental issue is the determination of its range of validity. However, many complex fluids exhibit a nonlinear response even at very small applied strains. This can provide an alternate means of probing the properties of the system; the strain amplitude, $|\gamma|$, can be used as an independent parameter $[2,3]$. While the nonlinear rheological properties of complex fluids are very diverse [1], most colloidal suspensions and polymer solutions show a simple yielding behavior, where both the storage $\left(G^{\prime}\right)$ and loss $\left(G^{\prime \prime}\right)$ modulus decrease monotonically as $|\gamma|$ increases. Less common is strain hardening (or strain-stiffening), where $G^{\prime}$ increases with $|\gamma|$. This can be observed in polymer solutions with strongly cross-linked chains [1], soft-biological tissues [4,5], and in dense colloidal suspensions [6,7]. There is another type of observed rheological behavior that lies between these two kinds of responses: as $|\gamma|$ increases, $G^{\prime}$ continuously decreases above a critical strain, whereas $G^{\prime \prime}$ exhibits a distinct peak before decreasing at even larger applied strains. This behavior is ubiquitous in metastable complex fluids. It was first reported in carbon black solutions [8]; many other systems exhibit similar behavior, including polymer systems [9], fumed silica [10,11], colloidal glasses $[12,13]$, emulsions $[14,15]$, gels [16] and electrorheological fluids $[17,18]$. Despite the

(c) EDP Sciences 
diversity of these systems, the observed behavior is strikingly universal: (i) The peak in $G^{\prime \prime}$ appears around $10^{-2} \lesssim|\gamma| \lesssim 1$ over a wide range of frequencies; (ii) the peak in $G^{\prime \prime}$ is observed for a frequency regime where the system is elastic, with $G^{\prime}>G^{\prime \prime}$; and (iii) the shear thinning exponent $\nu$ of $G^{\prime \prime} \propto|\gamma|^{-\nu}$ at larger strains is always about one half of the exponent associated with $G^{\prime}$. There have been many attempts to explain these universally observed phenomena. It has been argued that the increase of $G^{\prime \prime}$ as a function of $|\gamma|$ is due to the increase of the effective volume of the temporal network structure in polymer solutions [9]. Alternative suggestions include the breakdown of aggregates into small clusters which are more dissipative [11] and the rearrangement of clusters due to the strain associated with thickening [17]. The phenomenological soft glassy rheology (SGR) model also accounts for the peak in $G^{\prime \prime}$ as a function of $|\gamma|$, and posits that flow near the glass transition arises from activated barrier crossing [19]. However, a full microscopic account of this ubiquitous behavior is still lacking.

In this Letter, we show that the origin of the $|\gamma|$-dependent behavior of $G^{*}$ is in fact remarkably general and transcends the physical ingredients of the SGR picture; a peak in $G^{\prime \prime}$ should be present in any viscoelastic complex fluid that behaves like an elastic solid at small strain but yields and flows at larger strains. In particular, we explicitly demonstrate that this is true even in the absence of local activated processes. To make this argument more quantitative, we focus on colloidal suspensions in the supercooled state and calculate the $\omega$ and $|\gamma|$ dependence of $G^{*}$ using mode-coupling theory (MCT), generalized to account for the presence of oscillatory shear strain. This method successfully accounts for the peak observed in our experiments on a hard sphere colloidal suspension near its glass transition density.

The presence of the peak can be qualitatively explained using the following simple argument. Throughout the paper, an oscillatory strain, $\gamma(t)=|\gamma| \mathrm{e}^{i \omega t}$, is considered. In the small strain limit, the stress is written as $\sigma(t)=G^{*}(\omega) \gamma(t)$. As the simplest example, we consider a Maxwell model, where the storage and loss moduli are given by

$$
G^{\prime}(\omega) \propto \frac{\left(\omega \tau_{0}\right)^{2}}{1+\left(\omega \tau_{0}\right)^{2}} \quad \text { and } \quad G^{\prime \prime}(\omega) \propto \frac{\omega \tau_{0}}{1+\left(\omega \tau_{0}\right)^{2}},
$$

respectively. Here $\tau_{0}$ is a characteristic relaxation time which in many systems represents the structural relaxation time. According to this equation, $G^{\prime \prime}(\omega)$ has a peak around $\omega \simeq 1 / \tau_{0}$. By contrast, for large strains many complex fluids experience a drastic decrease of their structural relaxation time. Such a decrease can be characterized by the strain rate amplitude, $|\dot{\gamma}|$, and is given by the phenomenological expression

$$
1 / \tau \simeq 1 / \tau_{0}+K|\dot{\gamma}|^{\nu}
$$

where $\nu$ is a positive exponent and $K$ is a constant. If $\tau_{0}$ is replaced by $\tau$ in Eq. (10), a peak of $G^{\prime \prime}$ should be observed at $\omega \simeq 1 / \tau \simeq K|\dot{\gamma}|^{\nu}$; this equality is satisfied if $\omega \gg 1 / \tau_{0}$ and the system's intrinsic relaxation is very slow (thus $1 / \tau_{0}$ can be neglected). In particular, metastable systems which show slow dynamics, such as supercooled fluids and gels, have an exponent $\nu$ very close to unity and $K$ of order $1[20,21]$; then, the peak always appears near $\omega \simeq|\dot{\gamma}|$ or $|\gamma| \simeq 1$. This argument also accounts for the facts that (i) the position of the peak is relatively insensitive to the frequency, (ii) the peak of $G^{\prime \prime}$ should appear at frequencies where the linear viscoelasticity is dominantly elastic, $\left(G^{\prime}>G^{\prime \prime}\right)$, and (iii) the shear thinning exponents at larger strains are related and are given by $G^{\prime} \simeq|\gamma|^{-2 \nu}$ and $G^{\prime \prime} \simeq|\gamma|^{-\nu}$. Considering the broad diversity of systems, it is remarkable that this simple argument correctly captures the features of the peak in $G^{\prime \prime}$. The above argument is based on the assumption that nonlinear effects solely stem from the strain amplitude $|\gamma|$ (or more precisely from the strain 
rate amplitude $|\dot{\gamma}|=\omega|\gamma|)$ and are independent of the time-dependence of the strain. In other words, the stress is given by $\sigma(t)=G^{*}(\omega,|\gamma|) \gamma(t)$. This is consistent with recent experiments which have taken the effect of higher order harmonics into account and shown that they account for at most $10 \%$ of the response [3,22], thus, lending support to the notion these terms are negligible.

In order to make this argument more quantitative, we use a microscopic description based on MCT. First, we focus on a typical complex fluid which shows a peak in $G^{\prime \prime}$ as a function of $|\gamma|$, namely, a colloidal suspension in the supercooled state. To date, MCT is the only first principles theory that describes the dynamics of the supercooled state. Recently, several attempts have been made to extend the MCT approach to systems under shear strain for either stationary systems $[20,21,23]$ or for transient states $[24,25]$. In all previous analyses, $\dot{\gamma}$ has been assumed to be time-independent. Here we generalize the analysis $[20,21]$ to time-dependent oscillatory strain. We consider strain in the $x$ direction given by $\gamma(t)=|\gamma| \sin (\omega t)$. The arguments used are exactly the same as for the constant shear case [20,21], which should be valid as long as the shear rate amplitude $\omega|\gamma|$ is small compared to the inverse of the diffusion time $a^{2} / D$. We define a density correlation function which satisfies generalized translational invariance, $F(k, t)=N^{-1}\left\langle\delta \rho_{\mathbf{k}(-t)}(t) \delta \rho_{-\mathbf{k}}(0)\right\rangle$, where $N$ is the total particle number and $\mathbf{k}(t)$ is the time dependent wavevector defined as $\mathbf{k}(t)=\left(k_{x}, k_{y}+\gamma(t) k_{x}, k_{z}\right)$ [20]. Then, the MCT equation for $F(k, t)$ is

$$
\frac{\mathrm{d} F(\mathbf{k}, t)}{\mathrm{d} t}=-\frac{D k(-t)^{2}}{S(|\mathbf{k}(-t)|)} F(\mathbf{k}, t)-\int_{0}^{t} \mathrm{~d} t^{\prime} M\left(\mathbf{k}(-t), t-t^{\prime}\right) \frac{\mathrm{d} F\left(\mathbf{k}, t^{\prime}\right)}{\mathrm{d} t^{\prime}}
$$

where $D$ is the diffusion constant in the dilute limit, $k=|\mathbf{k}|$, and $S(k)$ is the (unperturbed) static structure factor. $M(\mathbf{k}, t)$ is the MCT memory kernel and is a nonlinear function of $F(k, t)$ and $S(k)$, whose explicit expression is given in Refs. [20,21]. Solving Eq. (3) numerically is a demanding task because of the anisotropic nature of the equation in the presence of the unidirectional strain. In order to make the computation tractable, we adopt the "isotropically sheared hard sphere model" (ISHSM) approximation proposed by Fuchs et al. [24], where the anisotropy of the strain which appears in $\mathbf{k}(t)$ is neglected and the scalar $|\mathbf{k}(t)|$ is approximated by $|\mathbf{k}(t)|=\sqrt{k^{2}+k_{x} k_{y} \gamma(t)+k_{y}^{2} \gamma^{2}(t)} \simeq \sqrt{1+\gamma^{2}(t) / 3} k$. This approximation is expected to be qualitatively correct as verified by the observation that the dynamics of supercooled liquids under shear strain is surprisingly isotropic [21]. We solve Eq. (33) using this approximation and evaluate $F(k, t)$ for a given set of $\omega$ and $|\gamma|$. The complex modulus is calculated in the same manner as $M(k, t)$ in eq.(3) and is given by [21]

$$
G_{\mathrm{NL}}^{*}(\omega,|\gamma|)=\frac{k_{\mathrm{B}} T i \omega}{2} \int_{0}^{\infty} \mathrm{d} t \int \frac{\mathrm{d} \mathbf{k}}{(2 \pi)^{3}} \mathrm{e}^{i \omega t} V(k) F^{2}(k, t) V(|\mathbf{k}(t)|),
$$

where $V(k)=k_{x} S^{-2}(k) n S^{\prime}(k)$ and $n$ is the particle number density. We calculate $G_{\mathrm{NL}}^{*}(\omega ; \gamma)$ for a hard sphere colloidal suspension with particle radius $a$ for a volume fraction $\phi=4 n \pi a^{3} / 3$ near the MCT glass transition point, $\phi_{c} \approx 0.516$, at which point $F(k, t)$ undergoes an ergodicnonergodic transition [26]. In Fig. 1, we show the frequency and strain dependence of $G_{\mathrm{NL}}^{*}(\omega,|\gamma|)$. The volume fraction is set to $\phi=\phi_{c} \times\left(1-10^{-3}\right)$. We calculate $S(k)$ from the Percus-Yevick equation. The frequency is scaled by $4 a^{2} / D$ and $G_{\mathrm{NL}}^{*}$ is plotted in units of $k_{\mathrm{B}} T / 8 a^{3}$. The behavior of $G_{\mathrm{NL}}^{\prime \prime}$ demonstrates that, in the small strain limit, a broad dip ( $\beta$-regime) is observed between $10^{-3} \lesssim \omega \lesssim 10^{1}$. A peak at low frequencies $\omega \simeq 10^{-4}$ is the $\alpha$-relaxation regime below which liquid-like relaxation is observed. In the $\beta$-regime, $G_{\mathrm{NL}}^{\prime}$ is larger than $G_{\mathrm{NL}}^{\prime \prime}$ and the system is solid-like. As the strain is increased in this regime, a weak 

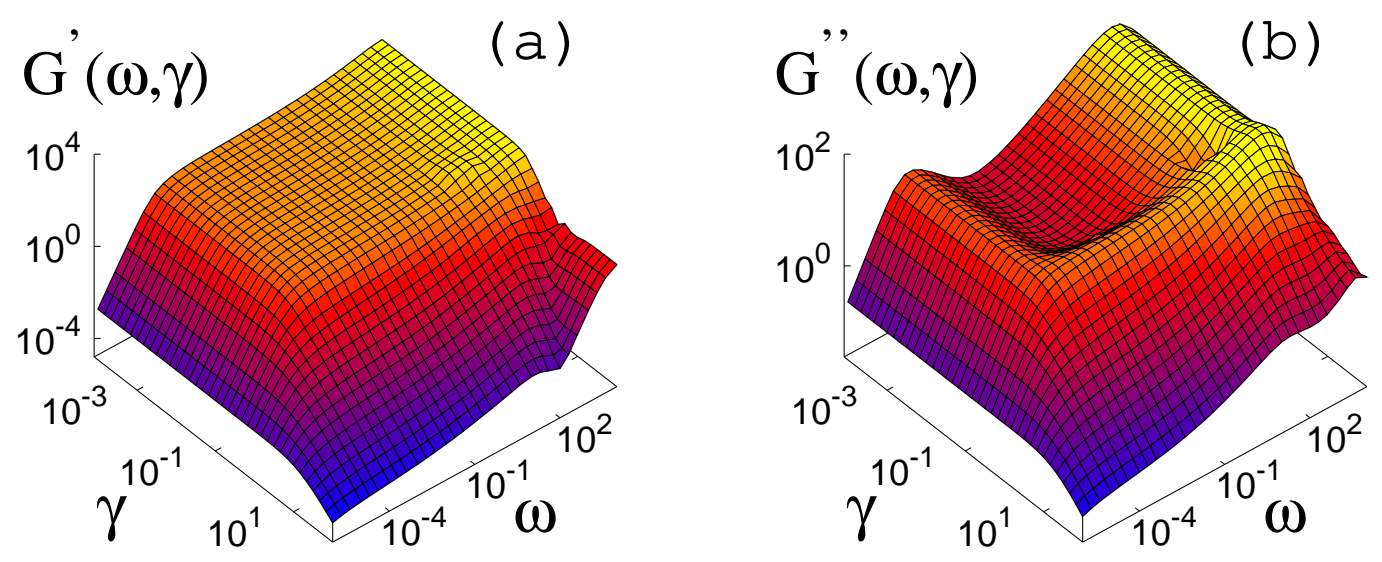

Fig. 1 - Frequency and strain dependence of (a) the storage modulus $G_{\mathrm{NL}}^{\prime}(\omega ; \gamma)$ and (b) the loss modulus $G_{\mathrm{NL}}^{\prime \prime}(\omega ; \gamma)$ for hard sphere suspension near the glass transition density.

peak in $G_{\mathrm{NL}}^{\prime \prime}$ is observed around $|\gamma| \simeq 0.4$, whereas $G_{\mathrm{NL}}^{\prime}$ starts to decrease monotonically. At even larger strains, $G_{\mathrm{NL}}^{\prime \prime}$ also starts to decrease. The decrease of $G_{\mathrm{NL}}^{*}$ is well described by a power law, $G_{\mathrm{NL}}^{\prime} \simeq|\gamma|^{-2 \nu}$ and $G_{\mathrm{NL}}^{\prime \prime} \simeq|\gamma|^{-\nu}$ with $\nu=0.9$. The features that appear at both high frequencies and strain amplitudes occur in the regime where $\omega|\gamma|$ (or the Péclet number $\left.\omega|\gamma| a^{2} / D\right)$ becomes very large and thus the validity of the present MCT framework is questionable. Thus, they should not be considered as physical. The position of the peak appears to be almost frequency independent. Although $\nu$ varies with the system, these general features are shared by most experiments where the peak is observed [8-18]. It should be emphasized however that the peak in $G_{\mathrm{NL}}^{\prime \prime}$ as a function of $|\gamma|$ is not generic, intrinsic shear thickening $[5,7]$ but originates instead from a strong decrease of the structural relaxation ( $\alpha$-relaxation) time at large strains. Indeed, the $\alpha$-relaxation time, $\tau_{\alpha}$, obtained from $F(k, t)$ is found to behave as $\tau_{\alpha} \simeq|\dot{\gamma}|^{-\nu}$. Furthermore, our results show that the origin of the peak in $G_{\mathrm{NL}}^{\prime \prime}$ is more general than that posited by the SGR model [19]. In particular, MCT does not capture the local rearrangements of particles that occur via activated barrier crossing, but it nevertheless properly captures the features of $G_{\mathrm{NL}}^{\prime \prime}$. We thus conclude that activated processes are not necessary to describe these features.

While these calculations have been done for oscillatory strain, we note that the timedependence of the generated strain, $\gamma(t)$, has only a small effect on the $\omega$ and $|\gamma|$ dependence of $G_{\mathrm{NL}}^{*}$. To show this, we calculate $G_{\mathrm{NL}}^{*}$ by approximating the oscillatory strain rate by a constant, $\dot{\gamma}(t) \simeq \omega|\gamma|$. $G_{\mathrm{NL}}^{*}$ thus obtained is qualitatively similar to that calculated for the case of oscillatory strain with a slight shift to a lower strain amplitude $(|\gamma| \approx 0.3)$ of the peak position. These conclusions support our qualitative argument based on the Maxwell model. The qualitative argument also asserts that $G^{*}(\omega)$ is a function of $\hat{\omega} \equiv \omega \tau$ (see Eq. (11) and the effect of the large strain comes in only through the relaxation time in the form of Eq. (2). We find that this argument is valid for structural relaxation in glassy systems within the approximate framework of the MCT described in this paper. $\tau_{\alpha}(\dot{\gamma})$ extracted from the 
peaks of $G_{\mathrm{NL}}^{\prime \prime}$ shown in Fig.1 (b) as a function of the strain rate is well described by Eq. (2) with $\tau_{0}=\tau_{\alpha}(\dot{\gamma} \rightarrow 0), \nu=0.9$ and a positive constant $K\left(\simeq 2.5\right.$ in units of $\left.4 a^{2} / D\right)$. We also find that, around the peaks, the shape of $G_{\mathrm{NL}}^{*}(\omega ; \gamma)$ as a function of $|\dot{\gamma}|$ matches well with $G^{*}\left(\omega \tau_{\alpha}\right)$ calculated at $|\dot{\gamma}|=0$ as a function of $\omega \tau_{\alpha}$, where the strain dependence of $\tau_{\alpha}$ is given by Eq. (2).

To demonstrate the power of the MCT approach, we make a quantitative comparison of theory with an experimental system, namely, a hard sphere colloidal suspension. Experiments are performed with sterically stabilized PMMA particles suspended in a mixture of decaline and cycloheptyle bromide. Their radius is $a=197 \mathrm{~nm}$ and their volume fraction is $\phi \approx 0.56$, which is a super-cooled fluid, just below the glass transition. Rheological experiments are performed in this supercooled state at room temperature using a strain-controlled rheometer (ARES, TA Instruments). Results for strain-sweep measurements at a fixed frequency $\omega=$ $0.2 \mathrm{rad} / \mathrm{sec}$ are shown in Fig. 2. A distinct peak is observed in $G_{\mathrm{NL}}^{\prime \prime}$ at $|\gamma| \approx 0.15$, while the onset of a monotonic decrease of $G_{\mathrm{NL}}^{\prime}$ is observed at the same strain amplitude. The shear thinning behavior is well described by a power law, with a shear thinning exponent $\nu \approx 0.7$. In the linear response regime where the strain is small, MCT is known to be successful at predicting the frequency dependence quantitatively and the overall shape of the complex modulus qualitatively near the glass transition points. Due to the generically approximate nature of $\mathrm{MCT}$, a direct comparison to experimental data still requires several fitting parameters. It is known that MCT underestimates the glass transition volume fraction by more than $10 \%[27]$ (experiments predict the glass transition density around $\phi_{g} \approx 0.58$, whereas MCT predicts the nonergodic transition at $\left.\phi_{c} \approx 0.516\right)$. MCT also underestimates the amplitude of the complex modulus over a wide range of frequencies, as theoretically shown for a semi-dilute suspension $[28,29]$. Moreover, MCT entirely neglects the effects of hydrodynamic interactions. In order to determine the parameters to correct for the abovementioned problems, we first perform a linear viscoelastic measurement for wide range of frequencies $\left(10^{-2} \leq \omega \leq 10^{2} \mathrm{rad} / \mathrm{sec}\right)$ in the small-strain limit $(|\gamma|=0.005$ and 0.01$)$. Then, we regard the volume fraction as a free parameter and determine it in such a way that the frequency dependence of $G_{\mathrm{NL}}^{*}(\omega,|\gamma|=0)$ calculated by MCT in the absence of strain matches with the shape of the measured $G^{*}(\omega,|\gamma| \ll 1)$. The volume fraction thus determined is $\phi=\phi_{c} \times\left(1-10^{-2.3}\right)$. The hydrodynamic interactions are approximately taken into account by replacing the diffusion constant $D$ in eq.(3) by $D_{\mathrm{HI}}(k)$, the short time collective diffusion coefficient with full hydrodynamic interactions, which was calculated using the BeenakkerMazur theory [30]. Finally, the amplitude of $G^{*}$ is matched with experimental data in the $\gamma \rightarrow 0$ limit. For these parameters, we calculate $G_{\mathrm{NL}}^{*}$ for finite strains. The results thus obtained reproduce the peak in $G_{\mathrm{NL}}^{\prime \prime}$ but overestimate the position of the peak. The lines in Fig. 2 are obtained by using the MCT calculations with a strain that is increased by a factor of approximately 3 , so that the peak overlaps with the experimental data. The discrepancy of the peak position might stem largely from the ISHSM approximation which we introduced for technical reasons. Indeed, it is obvious that the ISHSM approximation underestimates the onset of shear thinning because it neglects the term linear in $|\dot{\gamma}|$. The shear thinning after the peak exponent from MCT is found to be $\nu \simeq 0.9$ for $G_{\mathrm{NL}}^{\prime \prime}$ and $2 \nu$ for $G_{\mathrm{NL}}^{\prime}$. While MCT overestimates of the shear thinning exponent and the height and position of the peak which is somewhat larger than that extracted from experiment, the overall shape and qualitative behavior of $G_{\mathrm{NL}}^{*}$ is captured well. Considering the known problems associated with MCT even in the linear regime, the result shown in Fig. 2 generated by our "nonequilibrium" MCT is rather remarkable.

Although in this Letter we have investigated a limited class of systems where the MCT approach may be applicable, we believe that our analysis is more general and gives significant 


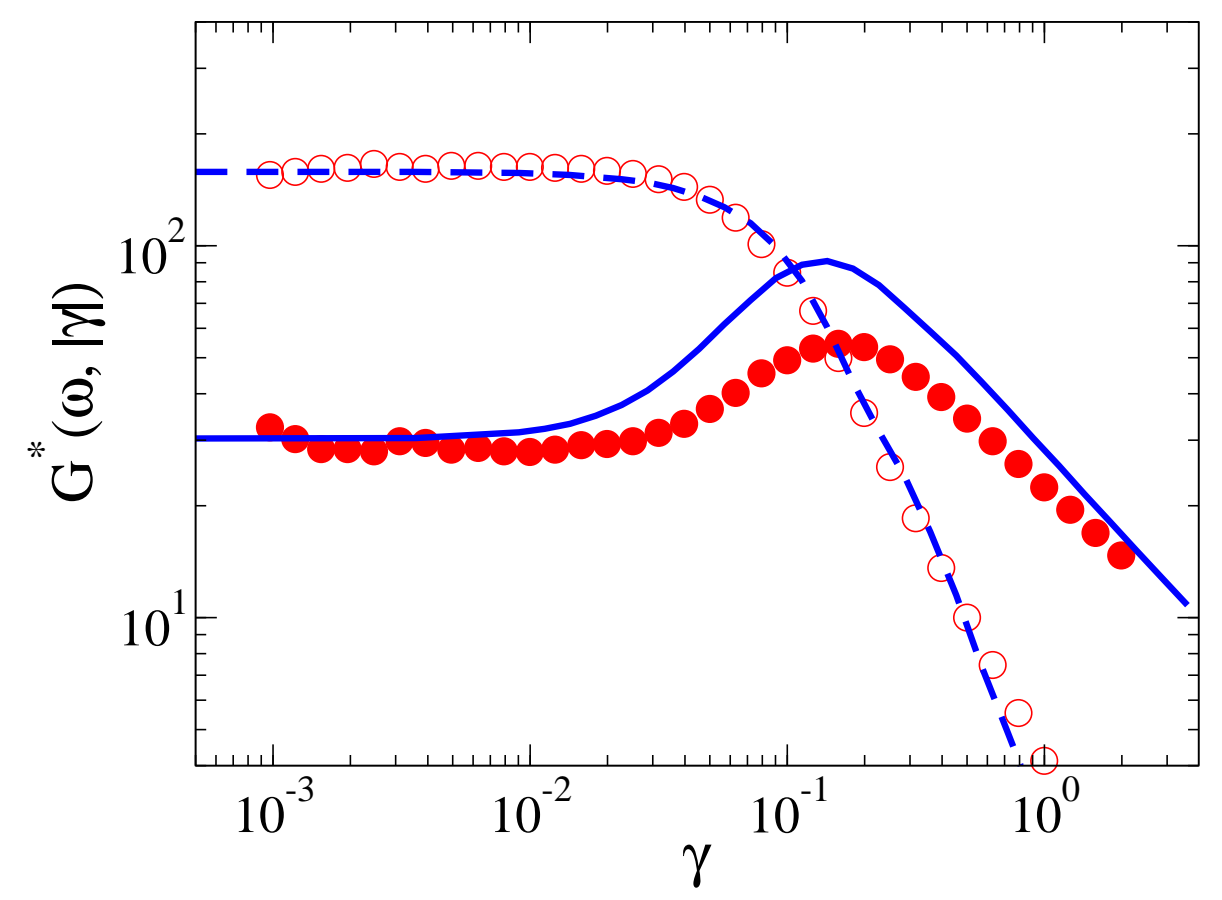

Fig. 2 - Comparison of MCT with experiment for a dense hard sphere suspension for $\omega=0.2$ $\mathrm{rad} / \mathrm{sec}$. The empty and filled circles represent the storage modulus, $G_{\mathrm{NL}}^{\prime}$, and loss modulus, $G_{\mathrm{NL}}^{\prime \prime}$, respectively. Bold lines represents the MCT results using the ISHSM approximation near the glass transition density. The lines are shifted in the direction of the strain by a factor of 3 . The modulus is plotted in units of $k_{\mathrm{B}} T / 8 a^{3}$.

insight into the underlying physics of the peak in the loss modulus as a function of strain, observed in various types of complex fluids. The major origin of the peak in the loss modulus is the strong decrease of the structural relaxation time at large strains, and has no connection whatsoever with intrinsic shear thickening. This peak should always be observed for systems which exhibit slow dynamics and possess a broad band of solid-like behavior above a crossover frequency where the storage modulus is larger than the loss modulus. The theoretical argument predicts that, near the peak of $G^{\prime \prime}$, the strain and frequency dependent viscoelasticity is well approximated by the linear viscoelasticity $G^{*}\left(\omega \tau_{\alpha}(|\dot{\gamma}|)\right)$, as a function of the frequency scaled by the relaxation time $\tau_{\alpha}$, whose strain dependence is given by Eq (2). This implies that nonlinear measurements are a promising alternative for probing the structure and dynamics of systems where linear viscoelastic experiments are limited to a narrow frequency window [31]. This will provide important insight into the physical origins of the nonlinear viscoelastic response of a wide range of soft materials.

The authors acknowledge support from the NSF (\# 0134969, KM and DRR), (DMR0602684, HMW and DAW), the Harvard MRSEC (DMR-0213805) and the Swiss National Science Foundation (HMW). 


\section{REFERENCES}

[1] R. G. Larson, The Structure and Rheology of Complex Fluids" (Oxford University Press, Oxford, 1999).

[2] W. Macosko, Rheology: Principle, Measurements, and Applications (VCH, New York, 1993).

[3] K. Hyun et al., J. Non-Cryst. Solids 107, 51 (2002).

[4] C. Gardel et al., Science 304, 1301 (2004).

[5] C. Storm et al., Nature 435, 191 (2005).

[6] E. Bertrand et al., Phys. Rev. E 66, 060401(R) (2002).

[7] J. R. Melrose and R. C. Ball, J. Rheol. 48, 937 (2004).

[8] A. R. Payne, J. Appl. Polym. Sci. 7, 873 (1963).

[9] V. Tirtaatmadja et al., Macromolecules 30, 1426 (1997a); ibid. 30, 3271 (1997b).

[10] S. R. Raghavan and S. A. Khan, J. Rheol. 39, 1311 (1995); J. Colloid and Interface Sci. 185, 57 (1997).

[11] F. Yziquel et al., Rheol. Acta 38, 14 (1999).

[12] T. G. Mason and D. A. Weitz, Phys. Rev. Lett. 75, 2770 (1995).

[13] G. Petekidis et al., Phys. Rev. E 66, 051402 (2002).

[14] T. G. Mason et al., Phys. Rev. Lett. 75, 2051 (1995).

[15] C. Bower et al., Rheol. Acta 38, 145 (1999).

[16] N. Altmann et al., J. Non-Newtonian Fluid Mech. 124, 129 (2004).

[17] M. Parthasarathy and D. J. Klingenberg, J. Non-Newtonian Fluid Mech. 81, 83 (1999).

[18] H. G. Sim et al., J. Rheol. 47, 879 (2003).

[19] P. Sollich, Phys. Rev. E 58, 738 (1998); P. Sollich et al., Phys. Rev. Lett. 78, 2020 (1997).

[20] K. Miyazaki and D. R. Reichman, Phys. Rev. E 66, 050501(R) (2002).

[21] K. Miyazaki et al., Phys. Rev. E 70, 011501 (2004).

[22] M. Wilhelm, Macromolecular Materials and Engineering 287, 83 (2002).

[23] G. Szamel, Phys. Rev. Lett. 93, 178301 (2004).

[24] M. Fuchs and M. E. Cates, Phys. Rev. Lett. 89, 248304 (2002); Faraday Discuss. 123, 267 (2003a); J. Phys.: Condens. Matter 15, S401 (2003b); ibid. 17, S1681 (2005).

[25] M. Fuchs and M. Ballauff, Colloids and Surfaces A 270-271, 232 (2005); J. Chem. Phys. 122, 094707 (2005b).

[26] W. Götze and L. Sjögren, Rep. Prog. Phys. 55, 241 (1992).

[27] W. van Megen and S. M. Underwood, Phys. Rev. Lett. 70, 2766 (1993): Phys. Rev. E 49, 4206 (1994).

[28] R. Verberg, I. M. de Schepper, and E. G. D. Cohen, J. Stat. Mech. 87, 1037 (1997).

[29] B. Cichocki and B. U. Felderhof, Phys. Rev. A 43, 5405 (1991).

[30] C. W. J. Beenakker and P. Mazur, Physica A 126, 349 (1984).

[31] H. Wyss et al., (unpublished). 